Mathematical Modelling and AnAlysis

Volume 16 Number 1, March 2011, 23-38

Doi:10.3846/13926292.2011.559449

(C) Vilnius Gediminas Technical University, 2011
www.tandf.co.uk/journals/TMMA

Publisher: Taylor\&Francis and VGTU

Online ISSN: 1648-3510

Print ISSN: 1392-6292

\title{
Two-Parameter Nonlinear Oscillations: The Neumann Problem
}

\author{
A. Gritsans and F. Sadyrbaev \\ Daugavpils University \\ Parades str. 1, LV-5400 Daugavpils, Latvia \\ E-mail(corresp.): armands.gricans@du.lv \\ E-mail: felix@latnet. $1 \mathrm{v}$
}

Received August 26, 2010; revised December 10, 2010; published online March 1, 2011

\begin{abstract}
Boundary value problems of the form $x^{\prime \prime}=-\lambda f\left(x^{+}\right)+\mu g\left(x^{-}\right)(i)$, $x^{\prime}(a)=0=x^{\prime}(b)$ (ii) are considered, where $\lambda, \mu>0$. In our considerations functions $f$ and $g$ are generally nonlinear. We give a description of a solution set of the problem $(i),(i i)$. It consist of all triples $(\lambda, \mu, \alpha)$ such that $(\lambda, \mu, x(t))$ nontrivially solves the problem $(i),(i i)$ and $\left|x^{\prime}(z)\right|=\alpha$ at zero points $z$ of the function $x(t)(i i i)$. We show that this solution set is a union of solution surfaces which are centro-affine equivalent. Each solution surface is associated with nontrivial solutions with definite nodal type. Properties of solution surfaces are studied. It is shown, in particular, that solution surface associated with solutions with exactly $i$ zeroes in the interval $(a, b)$ is centro-affine equivalent to a solution surface of the Dirichlet problem $(i)$, $x(a)=0=x(b),($ iii $)$ corresponding to solutions with odd number of zeros $2 j-1$ $(i \neq 2 j)$ in the interval $(a, b)$.
\end{abstract}

Keywords: Nonlinear oscillations, $\alpha$-spectrum, $\alpha$-branch, solution surfaces, solution curves, centro-affine equivalence, Neumann boundary value problem, Dirichlet boundary value problem.

AMS Subject Classification: 34B08; 34B15.

\section{Introduction}

Two-parameter nonlinear boundary value problems have been extensively studied in the literature, see for example $[1,2,3,5,8,11,14,16]$. Some of the mentioned references deal with the so called asymmetric oscillators. The simplest asymmetric oscillator is described by the Fučík differential equation $x^{\prime \prime}=-\lambda x^{+}+\mu x^{-}$, where $x^{+}=\max \{x, 0\}, x^{-}=\max \{-x, 0\}$. This equation possesses the property of positive homogeneity and any function $c x(t)$ solves it for $c \geq 0$ if $x(t)$ is a solution. The sum of two solutions $x_{1}(t)$ and $x_{2}(t)$ need not to be a solution.

A nonlinear asymmetric oscillator (in the meaning that restoring forces on the left and right sides nonlinearly depend on replacements $x(t)$ ) may be 
associated with equation $x^{\prime \prime}=-\lambda f\left(x^{+}\right)+\mu g\left(x^{-}\right)$, where $f$ and $g$ are nonlinear nonnegative functions. This equation with the Dirichlet boundary conditions was considered in $[4,5,7]$. In [4] the Dirichlet boundary value problem was investigated together with a normalization condition which is not needed in case of the Fučík equation since it fulfils automatically. The analytical description of the spectrum was obtained. The spectrum is a set of all pairs $(\lambda, \mu)$ such that the problem has a nontrivial solution. In [7] the Dirichlet problem was studied provided that one of the functions $f$ and $g$ is linear. In [5] some properties of the spectrum were analyzed. It was pointed out that branches of the spectrum may have separate components connected at infinity and even bounded separate components.

This article is continuation and expansion of [8], where the Neumann problem

$$
x^{\prime \prime}=-\lambda f\left(x^{+}\right)+\mu g\left(x^{-}\right), \quad x^{\prime}(a)=0=x^{\prime}(b),
$$

$a<b$, was considered. The main result in [8] is an analytical description of the spectrum provided in Theorem 1. In Definition 1 of the present article we substantially expand notion of a spectrum (a solution set is introduced) for the problem (1.1) and give full description of a spectrum in Theorem 4, thereby generalizing the main result in [8].

We consider the Neumann problem (1.1), where $\lambda$ and $\mu$ are positive parameters, and functions $f$ and $g$ satisfy the following conditions (we formulate these conditions only for a function $f$, supposing that analogous conditions are fulfilled for a function $g$ also):

(A1) $f$ is a $[0,+\infty) \rightarrow[0,+\infty)$ continuous function, $f(x)>0$ for all $x>0$ and $f(0)=0$;

(A2) a first zero function (a time map) $t_{f}(\alpha)$ to the Cauchy problem

$$
x^{\prime \prime}+f(x)=0, \quad x(a)=0, \quad x^{\prime}(a)=\alpha>0
$$

is a $(0,+\infty) \rightarrow(0,+\infty)$ continuous function.

Sometimes we need the additional technical requirements on $f$ function. These conditions are:

(A3) for some $k \in \mathbb{N}$ :

$$
f(0)=f^{\prime}(0+)=\cdots=f^{(k-1)}(0+)=0, \quad 0<f^{(k)}(0+) \leq+\infty ;
$$

(A4) $\frac{F(x)}{x^{\kappa-1}} \rightarrow+\infty$ as $x \rightarrow+\infty$, where $F(x)=\int_{0}^{x} f(s) d s$, and $\frac{f(x)}{x^{\kappa}}$ is bounded for $x$ large for some $\kappa \geq 1$.

Definition 1. A solution set of the problem (1.1) is a set $F$ of all triples $(\lambda, \mu, \alpha)$ such that $(\lambda, \mu, x(t))$ nontrivially solves the problem (1.1) and

$$
\left|x^{\prime}(z)\right|=\alpha
$$

at zeros of a solution $x(t)$. 
Remark 1. A solution of the equation $x^{\prime \prime}=-\lambda f\left(x^{+}\right)+\mu g\left(x^{-}\right)$is a $C^{2}$-function. Therefore $x^{\prime}(t)$ is continuous. If $z_{1}$ and $z_{2}$ are two consecutive zeros of $x(t)$ then it is known that $x^{\prime}\left(z_{1}\right)=-x^{\prime}\left(z_{2}\right)$ due to the fact that the graph of $x(t)$ between two consecutive zeros is symmetric with respect to the middle point (the condition (A1) is only needed). Thus the relation $\left|x^{\prime}(z)\right|=\alpha$ holds at any zero of a solution $x(t)$ for given $\alpha>0$.

Remark 2. The conditions (A1) and (A2) together are more general than those imposed on $f$ in [8]. The conditions (A3) and (A4) together ensure that the relations (2.3) are valid. One could require that (2.3) holds instead of (A3) and (A4) in statements below.

Theorem 4 (see Section 4) claims that the solution set is a union $F=$ $\bigcup_{i=1}^{\infty} F_{i}^{ \pm}$of solution surfaces. The solution surface $F_{i}^{+}$(resp.: $F_{i}^{-}$) describes all $C^{2}$-solutions of the problem (1.1) with exactly $i$ zeros in the interval $(a, b)$ and positive (resp.: negative) $x(a)$. Notion of a solution surface was introduced in [14] for the case of the Dirichlet boundary value problem.

The problem (1.1) with a normalization condition

$$
\left|x^{\prime}(z)\right|=\alpha \quad \text { at zero points } z \text { of } x(t)
$$

will be referred to as $\alpha$-normalized problem. We will call $\alpha$-spectrum $F(\alpha)$ of $\alpha$-normalized problem a set of all pairs $(\lambda, \mu)$ such that the $\alpha$-normalized problem is nontrivially solvable. Description of $\alpha$-spectrum is similar to that given in [8], where a normalization condition $\left|x^{\prime}(z)\right|=1$ was used: the $\alpha$ spectrum is a union $F(\alpha)=\bigcup_{i=1}^{\infty} F_{i}^{ \pm}(\alpha)$ of $\alpha$-branches. The $\alpha$-branch $F_{i}^{+}(\alpha)$ (resp.: $\left(F_{i}^{-}(\alpha)\right)$ is a set of all $(\lambda, \mu)$ such that there exists a $C^{2}$-solution of the $\alpha$-normalized problem which 1$)$ has exactly $i$ zeros in the interval $(a, b) ; 2) x(a)$ is positive (resp.: negative); 3) the condition (1.5) fulfils for some fixed $\alpha>0$ (so $x(t)$ is a nontrivial solution).

Remark 3. Actually $\alpha$-branches are projections of $\alpha$-level sets of solution surfaces to the $(\lambda, \mu)$-plane, henceforth we will identify these subsets if it would not lead to confusion.

The special cases of the problem (1.1) are:

1) one-parameter linear problem

$$
x^{\prime \prime}=-\lambda x \quad\left[\text { equivalently } x^{\prime \prime}=-\lambda x^{+}+\lambda x^{-}\right], \quad x^{\prime}(a)=0=x^{\prime}(b),
$$

where $f=g=x$ and $\lambda=\mu$;

2) two-parameter piece-wise linear problem for the Fučík equation

$$
x^{\prime \prime}=-\lambda x^{+}+\mu x^{-}, \quad x^{\prime}(a)=0=x^{\prime}(b),
$$

where $f=g=x$;

3) one-parameter nonlinear problem

$$
\left.x^{\prime \prime}=-\lambda h(x) \text { [equivalently } x^{\prime \prime}=-\lambda f\left(x^{+}\right)+\lambda g\left(x^{-}\right)\right], x^{\prime}(a)=0=x^{\prime}(b),
$$

where $\lambda=\mu$ and $h(x)=\left\{\begin{array}{ll}f(x), & x \geq 0, \\ -g(-x), & x \leq 0,\end{array}\right.$ or $f(x)=h^{+}(x)$ and $g(x)=$ $h^{-}(-x)$ for all $x \geq 0$. 


\section{Time Maps}

Let $T_{f}(\alpha, \lambda)$ be the first zero function (the time map) for the Cauchy problem

$$
x^{\prime \prime}+\lambda f(x)=0, \quad x(a)=0, x^{\prime}(a)=\alpha>0 .
$$

Theorem 1. If a function $f$ satisfies the conditions (A1) and (A2) then:

1. The time map $T_{f}(\alpha, \lambda)$ is a continuous function and

$$
T_{f}(\alpha, \lambda)=\frac{1}{\sqrt{\lambda}} t_{f}\left(\frac{\alpha}{\sqrt{\lambda}}\right), \quad \forall \alpha, \lambda>0 ;
$$

2. If additionally $T_{f}(\alpha, \lambda)$ is differentiable, then it is a solution of the first order nonhomogeneous partial differential equation

$$
\alpha \frac{\partial T_{f}}{\partial \alpha}(\alpha, \lambda)+2 \lambda \frac{\partial T_{f}}{\partial \lambda}(\alpha, \lambda)=-T_{f}(\alpha, \lambda)
$$

for any $\alpha, \lambda>0$ and as a consequence the function $T_{f}$ cannot have (positive) extrema;

3. For any $\alpha, \beta, \lambda>0$ the rescaling formula is valid

$$
T_{f}(\beta, \lambda)=\frac{\alpha}{\beta} T_{f}\left(\alpha, \lambda \frac{\alpha^{2}}{\beta^{2}}\right) ;
$$

4. If additionally (A3) and (A4) hold then for a fixed $\alpha>0$ :

$$
\lim _{\lambda \rightarrow 0+} T_{f}(\alpha, \lambda)=+\infty, \quad \lim _{\lambda \rightarrow+\infty} T_{f}(\alpha, \lambda)=0
$$

Proof. The assertions 1 and 2 can be proved as the respective assertions in [6].

3. For $\alpha, \beta, \lambda>0$ we have

$$
\begin{aligned}
T_{f}(\beta, \lambda) & =\frac{1}{\sqrt{\lambda}} t_{f}\left(\frac{\beta}{\sqrt{\lambda}}\right)=\frac{1}{\sqrt{\lambda}} t_{f}\left(\frac{\alpha}{\sqrt{\lambda}} \frac{\beta}{\alpha}\right)=\frac{1}{\sqrt{\lambda}} t_{f}\left(\frac{\alpha}{\sqrt{\lambda \alpha^{2} / \beta^{2}}}\right) \\
& =\frac{1}{\sqrt{\lambda \alpha^{2} / \beta^{2}}} \frac{\alpha}{\beta} t_{f}\left(\frac{\alpha}{\sqrt{\lambda \alpha^{2} / \beta^{2}}}\right)=\frac{\alpha}{\beta} T_{f}\left(\alpha, \lambda \frac{\alpha^{2}}{\beta^{2}}\right) .
\end{aligned}
$$

4. The second relation (2.3) can be proved like in Proposition 3.8 in [6]. Now let us prove the first relation (2.3). Suppose the conditions (A1), (A2) and(A4) hold. Consider the function $h(x)=x^{\kappa}, \kappa \geq 1$. Since $\frac{f(x)}{h(x)}$ is bounded for $x$ large then $\limsup _{x \rightarrow+\infty} \frac{f(x)}{h(x)} \leq \Omega<+\infty$. Therefore by Opial's comparison theorem [12, Theorem 11] one has $\liminf _{u \rightarrow+\infty} \frac{\tau_{f}(u)}{\tau_{h}(u)} \geq \frac{1}{\sqrt{\Omega}}$, where $\tau_{f}(u)=t_{f}(\sqrt{2 F(u)})$ is a time map function (a first zero function), depending on $u=x_{\max }=F^{-1}\left(\frac{\alpha^{2}}{2}\right)=\max _{\left[0 ; t_{f}(\alpha)\right]}|x(t)|$ and defined for all $u>0 ; \tau_{h}(u)$ has 
analogous meaning and calculations show that $\tau_{h}(u)=c u^{-(\kappa-1) / 2}, c>0$ for all $u>0$.

From (A1) and (A2), it follows that the function $F$ is strictly increasing in the interval $(0 ;+\infty)$, besides $F(x) \rightarrow+\infty$ as $x \rightarrow+\infty$ since the time map $t_{f}(\alpha)$ exists and is finite for any $\alpha>0$. One has for a fixed $\alpha>0$ that $\lambda \rightarrow 0+$ iff $v=F^{-1}\left(\frac{\alpha^{2}}{2 \lambda}\right) \rightarrow+\infty$ and

$$
\begin{aligned}
\lim _{\lambda \rightarrow 0+} T_{f}(\alpha, \lambda) & =\lim _{\lambda \rightarrow 0+} \frac{1}{\sqrt{\lambda}} t_{f}\left(\frac{\alpha}{\sqrt{\lambda}}\right)=\lim _{v \rightarrow+\infty} \frac{\sqrt{2 F(v)}}{\alpha} t_{f}(\sqrt{2 F(v)}) \\
& =\lim _{v \rightarrow+\infty} \frac{\sqrt{2 F(v)}}{\alpha} \tau_{f}(v)=\frac{\sqrt{2}}{\alpha} \lim _{v \rightarrow+\infty} \sqrt{F(v)} \tau_{h}(v) \frac{\tau_{f}(v)}{\tau_{h}(v)} \\
& =\frac{\sqrt{2} c}{\alpha} \lim _{v \rightarrow+\infty} \sqrt{\frac{F(v)}{v^{\kappa-1}}} \frac{\tau_{f}(v)}{\tau_{h}(v)}=+\infty
\end{aligned}
$$

Remark 4. If $f=x^{r}, r>0$, then $T_{f}(\alpha, \lambda)=c \alpha^{-\frac{r-1}{r+1}} \lambda^{-\frac{1}{r+1}}, c=2^{\frac{r}{r+1}}(r+$ $1)^{\frac{1}{r+1}} A \neq 0, A=\int_{0}^{1} \frac{d s}{\sqrt{1-s^{r+1}}}$, in linear case $r=1$ one has $T_{f}(\alpha, \lambda)=\frac{\pi}{\sqrt{\lambda}}$. For the nonlinearity $f$ both relations (2.3) are valid, so the conditions (A3) and (A4) fix rather generic limit behavior of time maps.

\section{$3 \alpha$-Spectrum of a Two-Parameter Nonlinear Oscillator}

The next theorem gives description of an $\alpha$-spectrum with arbitrary normalization $\alpha>0$.

Theorem 2. Let functions $f$ and $g$ satisfy the conditions (A1)-(A4). Then $\alpha$-spectrum $F(\alpha)$ of the $\alpha$-normalized problem (1.1), (1.5) is a union of $\alpha$ branches

$$
F_{i}^{ \pm}(\alpha)=\left\{(\lambda, \mu): T_{f}(\alpha, \lambda)+T_{g}(\alpha, \mu)=2(b-a) / i\right\} \quad(i \in \mathbb{N}) .
$$

Proof. The proof is similar to that of Theorem 1 in [8].

Now let us consider properties of an $\alpha$-spectrum.

Theorem 3. Suppose functions $f$ and $g$ satisfy the conditions (A1)-(A4) and let $F(\alpha)=\bigcup_{i=1}^{\infty} F_{i}^{ \pm}(\alpha)$ be the $\alpha$-spectrum of the $\alpha$-normalized problem (1.1), $(1.5)$.

1. $\alpha$-branches $F_{i}^{ \pm}(\alpha)$ are nonempty sets for any $\alpha>0$ and $i \in \mathbb{N}$.

2. $\alpha$-branches $F_{i}^{+}(\alpha)$ and $F_{i}^{-}(\alpha)$ coincide for $i \in \mathbb{N}$.

3. $\alpha$-branches $F_{i}^{ \pm}(\alpha)$ and $F_{j}^{ \pm}(\alpha)$ do not intersect unless $i=j$.

4. Suppose $T_{f}\left(\alpha, \lambda_{*}\right)=\frac{2(b-a)}{i}$ for some $\lambda_{*}>0$ and $i \in \mathbb{N}$. Then the $\alpha$ branch $F_{i}^{ \pm}(\alpha)$ has a vertical asymptote at $\lambda=\lambda_{*}$. 
5. Suppose $T_{g}\left(\alpha, \mu_{*}\right)=\frac{2(b-a)}{i}$ for some $\mu_{*}>0$ and $i \in \mathbb{N}$. Then the $\alpha$ branch $F_{i}^{ \pm}(\alpha)$ has a horizontal asymptote at $\mu=\mu_{*}$.

6. Suppose the functions $\gamma t_{f}(\gamma)$ and $\gamma t_{g}(\gamma)$ are strictly increasing. For given $\alpha>0$ and $i \in \mathbb{N}$ the $\alpha$-branch $F_{i}^{ \pm}(\alpha)$ is a graph of strictly decreasing function $\mu=T_{g}^{-1}\left(\alpha, \frac{2(b-a)}{i}-T_{f}(\alpha, \lambda)\right)$.

7. For given $i \neq j$ and $\alpha>0$ the branches $F_{i}^{ \pm}(\alpha)$ and $F_{j}^{ \pm}\left(\frac{j}{i} \alpha\right)$ are centroaffine [13] equivalent under the mapping $\Omega_{i, j}: \mathbb{R}^{2} \rightarrow \mathbb{R}^{2},(\lambda, \mu) \stackrel{\Omega_{i, j}}{\longmapsto}$ $(\bar{\lambda}, \bar{\mu})$, where $\bar{\lambda}=\left(\frac{j}{i}\right)^{2} \lambda, \bar{\mu}=\left(\frac{j}{i}\right)^{2} \mu$.

8. Suppose $f=g$ and the function $\gamma t_{f}(\gamma)$ has a point of strict maximum (there is also a point of strict minimum, in view of (2.3)). Then there exists a normalization $\beta_{j}(j \in \mathbb{N})$ such that the branch $F_{j}^{ \pm}\left(\beta_{j}\right)$ has a separate bounded component.

Proof. Statements 1, 4 and 5 are valid due to the assertions 1 and 4 of Theorem 1. Statements 2 and 3 follow from (3.1).

6. If functions $\gamma t_{f}(\gamma)$ and $\gamma t_{g}(\gamma)$ are strictly increasing then functions $T_{f}(\alpha, \lambda)$ and $T_{g}(\alpha, \mu)$ strictly decrease from $+\infty$ to zero (recall $(2.3)$ ) as $\lambda \in$ $(0,+\infty)$ and $\mu \in(0,+\infty)$ respectively for a fixed normalization $\alpha>0$. For given $\alpha>0$ and $i \in \mathbb{N}$ due to the assertions 1 and 4 of Theorem 1 we can find unique $\lambda_{*}>0$ and $\mu_{*}>0$ such that $T_{f}\left(\alpha, \lambda_{*}\right)=\frac{2(b-a)}{i}$ and $T_{g}\left(\alpha, \mu_{*}\right)=\frac{2(b-a)}{i}$. Hence the branch $F_{i}^{ \pm}(\alpha)$ actually is the graph of the strictly increasing function $\mu=T_{g}^{-1}\left(\alpha, \frac{2(b-a)}{i}-T_{f}(\alpha, \lambda)\right)$ with the domain of definition $\left(\lambda_{*},+\infty\right)$ and the range of values $\left(\mu_{*},+\infty\right)$. Besides it has the vertical asymptote at $\lambda=\lambda_{*}$ and the horizontal asymptote at $\mu=\mu_{*}$, see points 4 and 5 of the theorem. Similar approach was realized to prove Theorem 2 in [5].

7. For given $i \neq j$ and $\alpha>0$ find $\bar{\alpha}=j \alpha / i$. Suppose $(\lambda, \mu) \in F_{i}^{ \pm}(\alpha)$, that is,

$$
i T_{f}(\alpha, \lambda)+i T_{g}(\alpha, \mu)=2(b-a) .
$$

Applying the rescaling formula (2.2) to the previous equation one gets

$$
\begin{aligned}
& i \frac{\bar{\alpha}}{\alpha} T_{f}\left(\bar{\alpha}, \lambda \frac{\bar{\alpha}^{2}}{\alpha^{2}}\right)+i \frac{\bar{\alpha}}{\alpha} T_{g}\left(\bar{\alpha}, \mu \frac{\bar{\alpha}^{2}}{\alpha^{2}}\right)=2(b-a), \\
& j T_{f}\left(\bar{\alpha},\left(\frac{j}{i}\right)^{2} \lambda\right)+j T_{g}\left(\bar{\alpha},\left(\frac{j}{i}\right)^{2} \mu\right)=2(b-a), \\
& j T_{f}(\bar{\alpha}, \bar{\lambda})+j T_{g}(\bar{\alpha}, \bar{\mu})=2(b-a),
\end{aligned}
$$

therefore $(\bar{\lambda}, \bar{\mu}) \in F_{j}^{ \pm}(\bar{\alpha})$. Since $\Omega_{i, j}^{-1}=\Omega_{j, i}$ one has $\Omega_{i, j}\left(F_{i}^{ \pm}(\alpha)\right)=F_{j}^{ \pm}(\bar{\alpha})$ and the branches $F_{i}^{ \pm}(\alpha)$ and $F_{j}^{ \pm}(\bar{\alpha})$ are centro-affine equivalent under the mapping $\Omega_{i, j}$.

8. Let $\gamma_{1}>0$ be a point of strict maximum of the function $\gamma t_{f}(\gamma)$. Then the function $\frac{1}{\sqrt{\lambda}} t_{f}\left(\frac{1}{\sqrt{\lambda}}\right)$ has strict maximum at $\lambda_{1}=1 / \gamma_{1}^{2}$. This corresponds to the normalization $\alpha=1$. In other words, $\frac{1}{\sqrt{\lambda_{1}}} t_{f}\left(\frac{1}{\sqrt{\lambda_{1}}}\right)=T_{f}\left(1, \lambda_{1}\right)$ and 
the function $T_{f}(1, \lambda)$ has a strict maximum at $\lambda=\lambda_{1}$. By virtue of $(2.2)$, $T_{f}(\beta, \lambda)=\frac{1}{\beta} T_{f}\left(1, \frac{\lambda}{\beta^{2}}\right)$.

Choose $\beta=\beta_{1}$ so that $\frac{1}{\beta_{1}} T_{f}\left(1, \lambda_{1}\right)=b-a$, this is possible due to the assertions 1 and 4 of Theorem 1 . Then $T_{f}\left(\beta_{1}, \lambda\right)$ has a strict maximum at $\lambda=$ $\lambda_{1} \beta_{1}^{2}$ and the corresponding maximal value is $b-a$. Then the sum $T_{f}\left(\beta_{1}, \lambda\right)+$ $T_{f}\left(\beta_{1}, \mu\right)$ has a point of strict maximum at $\left(\lambda_{1}, \lambda_{1}\right)$ and the corresponding maximal value is $(b-a)+(b-a)=2(b-a)$. In neighbouring points $(\lambda, \mu)$ one has that $T_{f}\left(\beta_{1}, \lambda\right)+T_{f}\left(\beta_{1}, \mu\right)<2(b-a)$. Evidently the branch $F_{1}^{ \pm}\left(\beta_{1}\right)$ has a bounded separate component (actually a point) at $\lambda=\mu=\lambda_{1}$. Changing $\beta$ further this component becomes larger.

The case when the function $\gamma t_{f}(\gamma)$ has a point of strict minimum can be considered analogously. We have proved that there exists a normalization $\beta_{1}>$ 0 such that the branch $F_{1}^{ \pm}\left(\beta_{1}\right)$ has a separate bounded component. Then the branch $F_{j}^{ \pm}\left(\beta_{j}\right)$ with the normalization $\beta_{j}=j \beta_{1}(j \in \mathbb{N})$ is centro-affine equivalent to the branch $F_{1}^{ \pm}\left(\beta_{1}\right)$, see assertion 7 of the theorem, and therefore has a separate bounded component too.

Remark 5. By the assertions 1 and 4 of Theorem 1:

$$
\lim _{\gamma \rightarrow 0+} \gamma t_{f}(\gamma)=0, \quad \lim _{\gamma \rightarrow+\infty} \gamma t_{f}(\gamma)=+\infty
$$

If the function $\gamma t_{f}(\gamma)$ is non constant in any interval $\left(\gamma_{1}, \gamma_{2}\right) \subset(0,+\infty)$ then existence of the strict maximum implies existence of the strict minimum and vice versa. The authors have no example of a nonlinearity $f$ satisfying the conditions (A1) $-(\mathbf{A} 4)$ such that the function $\gamma t_{f}(\gamma)$ is constant in some interval $\left(\gamma_{1}, \gamma_{2}\right) \subset(0,+\infty)$.

Remark 6. 1) An $i$-th $\alpha$-branch of the $\alpha$-spectrum of the $\alpha$-normalized one-parameter linear problem (1.6), (1.5) is the same set for all normalizations $\alpha>0$ and actually can be identified with the $i$-th spectral value of the linear oscillator: $\lambda_{i}=\left(\frac{\pi i}{b-a}\right)^{2}$. 2) An $i$-th $\alpha$-branch of the $\alpha$-spectrum of the $\alpha$-normalized two-parameter piece-wised linear problem (1.7), (1.5) is the same set for all normalizations $\alpha>0$ and coincide with the $i$-th branch of the classical Fučík spectrum $[3,8]: F_{i}^{ \pm}(\alpha)=\left\{(\lambda, \mu): \frac{\pi}{\sqrt{\lambda}}+\frac{\pi}{\sqrt{\mu}}=\frac{2(b-a)}{i}\right\}$. For continuation of this remark see Remark 14.

Remark 7. The centro-affine mapping $\Omega_{i, j}(i \neq j)$ preserves areas with the coefficient $J\left(\Omega_{i, j}\right)=(j / i)^{4}$ and has the inverse mapping $\Omega_{i, j}^{-1}=\Omega_{j, i}$. If $\left(\lambda_{1}, \mu_{1}\right) \in F_{1}^{ \pm}\left(\alpha_{1}\right)$ then the point $\left(j^{2} \lambda_{1}, j^{2} \mu_{1}\right)(j \in \mathbb{N}, j \neq 1)$ is a cross point of the branch $F_{j}^{ \pm}\left(j \alpha_{1}\right)$ with the line $\mu=\frac{\mu_{1}}{\lambda_{1}} \lambda$.

Example 1. In Fig. 1, branches $F_{1}^{ \pm}(1)$ and $F_{2}^{ \pm}(2)$ of the problem (1.1), (1.5) are depicted, where $f=g=x^{\frac{1}{3}}+x^{32}$ and $b-a=2.5$, and these branches are centro-affine equivalent.

Proposition 1. For given $i \neq j$ branches $F_{i}^{ \pm}(\alpha)$ and $F_{j}^{ \pm}(\beta)$ of spectra with different normalizations 


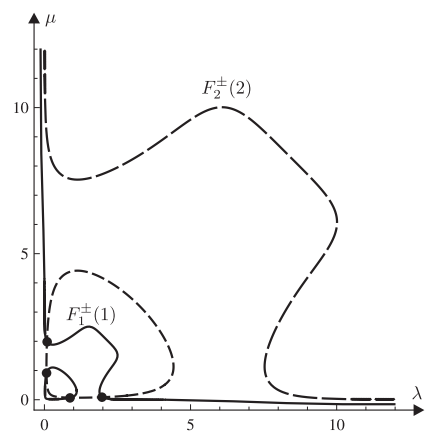

Figure 1. The case $f=g=x^{\frac{1}{3}}+x^{32}, b-a=2.5$. Numerical calculations show that the branches $F_{1}^{ \pm}(1)$ and $F_{2}^{ \pm}(2)$ of the problem (1.1), (1.5) with $\alpha=1$ (full line) and $\alpha=2$ (dashed line) respectively do not coincide and have 4 points of intersection.

- may coincide (in this case at least one of the functions $f$ and $g$ must be nonlinear), for example, if $f=g=x^{r}, r \neq 1$ and $\frac{\alpha}{\beta}=\left(\frac{j}{i}\right)^{-\frac{r+1}{r-1}}$,

- may do not intersect, for example, if 1) $f=g=x^{r}, r \neq 1$ and $\frac{\alpha}{\beta} \neq$ $\left.\left(\frac{j}{i}\right)^{-\frac{r+1}{r-1}}, 2\right) f=g=x$,

- may have points of intersection being distinct; see Fig. 1.

Proof. Suppose $i \neq j$ and two different normalizations $\alpha, \beta>0$ are given. To determine common points of the branches $F_{i}^{ \pm}(\alpha)$ and $F_{j}^{ \pm}(\beta)$ we need to solve the system

$$
\left\{\begin{array}{l}
T_{f}(\alpha, \lambda)+T_{g}(\alpha, \mu)=2(b-a) / i, \\
T_{f}(\beta, \lambda)+T_{g}(\beta, \mu)=2(b-a) / j
\end{array}\right.
$$

with respect to $(\lambda, \mu)$. If $f=g=x^{r}, r>0$, then using Remark 4 the system (3.3) becomes

$$
\left\{\begin{array}{l}
\alpha^{-\frac{r-1}{r+1}}\left(\lambda^{-\frac{1}{r+1}}+\mu^{-\frac{1}{r+1}}\right)=\frac{2(b-a)}{c i} \\
\beta^{-\frac{r-1}{r+1}}\left(\lambda^{-\frac{1}{r+1}}+\mu^{-\frac{1}{r+1}}\right)=\frac{2(b-a)}{c j} .
\end{array}\right.
$$

If $r \neq 1$ and $\frac{\alpha}{\beta}=\left(\frac{j}{i}\right)^{-\frac{r+1}{r-1}}$ the equations of the system (3.4) are equivalent, hence the branches $F_{i}^{ \pm}(\alpha)$ and $F_{j}^{ \pm}(\beta)$ coincide. If $r \neq 1$ and $\frac{\alpha}{\beta} \neq\left(\frac{j}{i}\right)^{-\frac{r+1}{r-1}}$ or $r=1$ (linear case) the system (3.4) has no solutions and the branches $F_{i}^{ \pm}(\alpha)$ and $F_{j}^{ \pm}(\beta)$ do not intersect. Thereby, if the branches $F_{i}^{ \pm}(\alpha)$ and $F_{j}^{ \pm}(\beta)$ with $\alpha \neq \beta$ and $i \neq j$ coincide then at least one of the functions $f$ and $g$ must be nonlinear.

Branches $F_{i}^{ \pm}(\alpha)$ and $F_{j}^{ \pm}(\beta)$ of spectra with $i \neq j$ and different normalizations $\alpha, \beta>0$ may be different with some points of intersection, see Fig. 1 . 


\section{Solution Set of a Two-Parameter Nonlinear Oscillator}

The next theorem gives description of a solution set of the problem (1.1).

Theorem 4. Let functions $f$ and $g$ satisfy the conditions (A1)-(A4). Then solution set $F$ of the problem (1.1) is a union of solution surfaces

$$
F_{i}^{ \pm}=\left\{(\lambda, \mu, \alpha): T_{f}(\alpha, \lambda)+T_{g}(\alpha, \mu)=2(b-a) / i\right\} \quad(i \in \mathbb{N}) .
$$

The solution surfaces have the following properties.

1. Solution surfaces $F_{i}^{ \pm}$are nonempty sets for any $i \in \mathbb{N}$.

2. Solution surfaces $F_{i}^{+}$and $F_{i}^{-}$coincide for $i \in \mathbb{N}$.

3. Solution surfaces $F_{i}^{ \pm}$and $F_{j}^{ \pm}$do not intersect unless $i=j$.

4. For given $i \neq j$ the solution surfaces $F_{i}^{ \pm}$and $F_{j}^{ \pm}$are centro-affine equivalent under the mapping $\Phi_{i, j}: \mathbb{R}^{3} \rightarrow \mathbb{R}^{3},(\lambda, \mu, \alpha) \stackrel{\Phi_{i, j}}{\longmapsto}(\bar{\lambda}, \bar{\mu}, \bar{\alpha})$, where $\bar{\lambda}=(j / i)^{2} \lambda, \bar{\mu}=(j / i)^{2} \mu, \bar{\alpha}=j \alpha / i$.

Proof. In view of Remark 3 one can regard an $i$-th solution surface as a union of $\alpha$-branches: $F_{i}^{ \pm}=\bigcup_{\alpha>0} F_{i}^{ \pm}(\alpha)$, hence the first statement follows from Theorem 2. The proof of properties 1 to 4 follows from Theorem 3 taking in mind Remark 3.

Remark 8. The centro-affine mapping $\Phi_{i, j}(i \neq j)$ preserves volumes with the coefficient $J\left(\Phi_{i, j}\right)=(j / i)^{5}$ and have the inverse mapping $\Phi_{i, j}^{-1}=\Phi_{j, i}$. If $\left(\lambda_{1}, \mu_{1}, \alpha_{1}\right) \in F_{1}^{ \pm}$then the point $\left(j^{2} \lambda_{1}, j^{2} \mu_{1}, j \alpha_{1}\right)(j \in \mathbb{N}, j \neq 1)$ is a cross point of the following subsets: 1) the solution surface $F_{j}^{ \pm}$and the parabolic cylinders $\lambda=\frac{\lambda_{1}}{\alpha_{1}^{2}} \alpha^{2}$ and $\mu=\frac{\mu_{1}}{\alpha_{1}^{2}} \alpha^{2}$, or equivalently 2) the solution surface $F_{j}^{ \pm}$, the parabolic cylinder $\lambda=\frac{\lambda_{1}}{\alpha_{1}^{2}} \alpha^{2}$ and the plane $\mu=\frac{\mu_{1}}{\lambda_{1}} \lambda$.

Example 2. If $f=g=x^{r}, r>0, r \neq 1$ then the solution surface $F_{i}^{ \pm}$is the graph of a two argument function $\alpha=\left(2(b-a) / i c\left(\lambda^{-\frac{1}{r+1}}+\mu^{-\frac{1}{r+1}}\right)\right)^{-\frac{r+1}{r-1}}$, see Remark 4.

Example 3. In Figs. 2 and 3 the visual examples of solution surfaces are presented.

Theorem 5. Let functions $f$ and $g$ satisfy the conditions (A1)-(A4). For given $i \neq 2 j$ the solution surface $F_{i}^{ \pm}$of the Neumann problem (1.1) is centroaffine equivalent to the solution surface

$$
\mathcal{F}_{2 j-1}^{ \pm}=\left\{(\lambda, \mu, \alpha): T_{f}(\alpha, \lambda)+T_{g}(\alpha, \mu)=(b-a) / j\right\}
$$

of the Dirichlet problem

$$
x^{\prime \prime}=-\lambda f\left(x^{+}\right)+\mu g\left(x^{-}\right), \quad x(a)=0=x(b)
$$

under the mapping $\Phi_{i, 2 j}: \mathbb{R}^{3} \rightarrow \mathbb{R}^{3},(\lambda, \mu, \alpha) \stackrel{\Phi_{i, 2 j}}{\longmapsto}(\widehat{\lambda}, \widehat{\mu}, \widehat{\alpha})$, where $\widehat{\lambda}=\left(\frac{2 j}{i}\right)^{2} \lambda$, $\widehat{\mu}=\left(\frac{2 j}{i}\right)^{2} \mu, \widehat{\alpha}=\frac{2 j}{i} \alpha$. If $i=2 j$ then $F_{i}^{ \pm}=\mathcal{F}_{2 j-1}^{ \pm}\left(F_{2}^{ \pm}=\mathcal{F}_{1}^{ \pm}, F_{4}^{ \pm}=\mathcal{F}_{3}^{ \pm}, \ldots\right)$. 

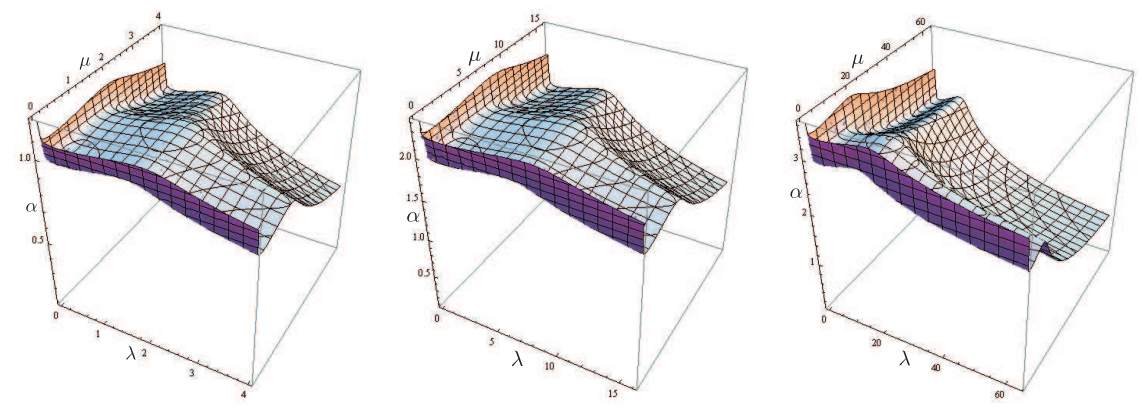

Figure 2. Centro-affine equivalent solution surfaces $F_{i}^{ \pm}(i=1,2,3$ from the left) of the problem (1.1), where $f=g=x^{2}+x^{32}$ and $b-a=2.5$.
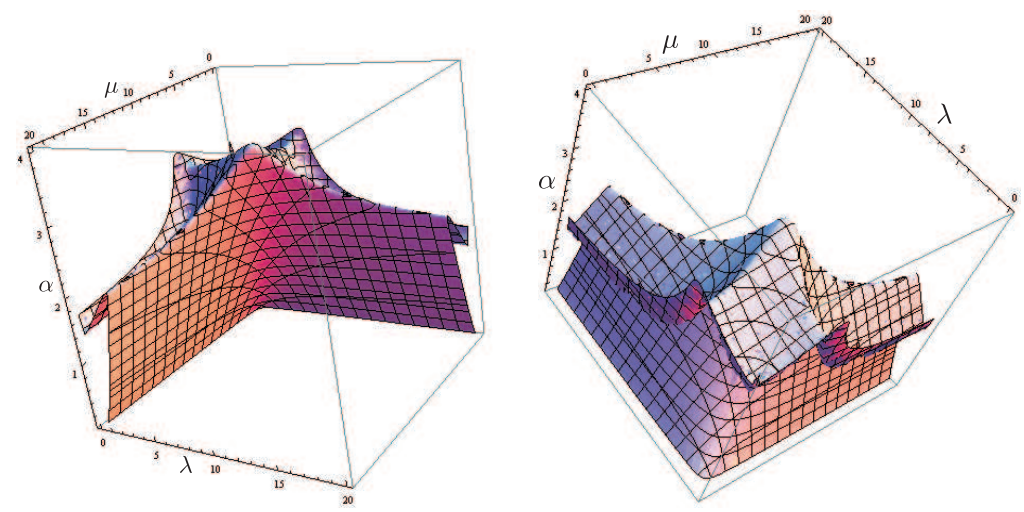

Figure 3. Two views of the solution surface $F_{1}^{ \pm}$of the problem (1.1), where $f=g=x^{\frac{1}{3}}+x^{41}$ and $b-a=1$.

Proof. Consider $i \neq 2 j$. Suppose $(\lambda, \mu, \alpha) \in F_{i}^{ \pm}(\alpha)$ :

$$
i T_{f}(\alpha, \lambda)+i T_{g}(\alpha, \mu)=2(b-a) .
$$

If $\widehat{\alpha}=\frac{2 j}{i} \alpha$ then applying the rescaling formula (2.2) to the previous equation one has

$$
\begin{aligned}
& i \frac{\widehat{\alpha}}{\alpha} T_{f}\left(\widehat{\alpha}, \lambda \frac{\widehat{\alpha}^{2}}{\alpha^{2}}\right)+i \frac{\widehat{\alpha}}{\alpha} T_{g}\left(\widehat{\alpha}, \mu \frac{\widehat{\alpha}^{2}}{\alpha^{2}}\right)=2(b-a), \\
& 2 j T_{f}\left(\widehat{\alpha},\left(\frac{2 j}{i}\right)^{2} \lambda\right)+2 j T_{g}\left(\widehat{\alpha},\left(\frac{2 j}{i}\right)^{2} \mu\right)=2(b-a), \\
& j T_{f}(\widehat{\alpha}, \widehat{\lambda})+j T_{g}(\widehat{\alpha}, \widehat{\mu})=b-a,
\end{aligned}
$$


hence $(\widehat{\lambda}, \widehat{\mu}, \widehat{\alpha}) \in \mathcal{F}_{2 j-1}^{ \pm}$. Since $\Phi_{i, 2 j}^{-1}=\Phi_{2 j, i}$ one obtains that $\Phi_{i, 2 j}\left(F_{i}^{ \pm}\right)=$ $\mathcal{F}_{2 j-1}^{ \pm}$and the solution surfaces $F_{i}^{ \pm}$and $\mathcal{F}_{2 j-1}^{ \pm}$are centro-affine equivalent under the mapping $\Phi_{i, 2 j}$.

Corollary 1. If $i \neq j$ the solution surface $\mathcal{F}_{2 i-1}^{ \pm}$is centro-affine equivalent to the solution surface $\mathcal{F}_{2 j-1}^{ \pm}$under the mapping $\Phi_{i, j}$.

Remark 9. The centro-affine mapping $\Phi_{i, 2 j}(i \neq 2 j)$ preserves volumes with the coefficient $J\left(\Phi_{i, 2 j}\right)=32(j / i)^{5}$ and has the inverse mapping $\Phi_{i, 2 j}^{-1}=\Phi_{2 j, i}$. If $\left(\lambda_{1}, \mu_{1}, \alpha_{1}\right) \in F_{1}^{ \pm}$then the point $\left(4 j^{2} \lambda_{1}, 4 j^{2} \mu_{1}, 2 j \alpha_{1}\right)(j \in \mathbb{N})$ is a cross point of the following subsets: 1$)$ the solution surface $\mathcal{F}_{2 j-1}^{ \pm}$and the parabolic cylinders $\lambda=\frac{\lambda_{1}}{\alpha_{1}^{2}} \alpha^{2}$ and $\mu=\frac{\mu_{1}}{\alpha_{1}^{2}} \alpha^{2}$, or equivalently 2) the solution surface $\mathcal{F}_{2 j-1}^{ \pm}$, the parabolic cylinder $\lambda=\frac{\lambda_{1}}{\alpha_{1}^{2}} \alpha^{2}$ and the plane $\mu=\frac{\mu_{1}}{\lambda_{1}} \lambda$.

One can prove the following Theorem in a similar manner having in mind assertion 7 of Theorem 3 and Theorem 5 .

Theorem 6. Let functions $f$ and $g$ satisfy the conditions (A1)-(A4). For given $i \neq 2 j$ and $\alpha>0$ the branch $F_{i}^{ \pm}(\alpha)$ of the $\alpha$-normalized Neumann problem (1.1), (1.5) is centro-affine equivalent to the branch

$$
\mathcal{F}_{2 j-1}^{ \pm}(2 j \alpha / i)=\left\{(\lambda, \mu, \alpha): T_{f}(\alpha, \lambda)+T_{g}(\alpha, \mu)=(b-a) / j\right\}
$$

of the $\left(\frac{2 j}{i} \alpha\right)$-normalized Dirichlet problem

$$
x^{\prime \prime}=-\lambda f\left(x^{+}\right)+\mu g\left(x^{-}\right), \quad x(a)=0=x(b)
$$

under the mapping $\Omega_{i, 2 j}: \mathbb{R}^{2} \rightarrow \mathbb{R}^{2},(\lambda, \mu) \stackrel{\Omega_{i, 2 j}}{\longmapsto}(\widehat{\lambda}, \widehat{\mu})$, where $\widehat{\lambda}=\left(\frac{2 j}{i}\right)^{2} \lambda$, $\widehat{\mu}=\left(\frac{2 j}{i}\right)^{2} \mu$. If $i=2 j$ then $F_{i}^{ \pm}(\alpha)=\mathcal{F}_{2 j-1}^{ \pm}(\alpha)\left(F_{2}^{ \pm}(\alpha)=\mathcal{F}_{1}^{ \pm}(\alpha), F_{4}^{ \pm}(\alpha)=\right.$ $\left.\mathcal{F}_{3}^{ \pm}(\alpha), \ldots\right)$.

Corollary 2. If $i \neq j$ the $\alpha$-branch $\mathcal{F}_{2 i-1}^{ \pm}(\alpha)$ is centro-affine equivalent to the $\left(\frac{j}{i} \alpha\right)$-branch $\mathcal{F}_{2 j-1}^{ \pm}\left(\frac{j}{i} \alpha\right)$ under the mapping $\Omega_{i, j}$.

Remark 10. The centro-affine mapping $\Omega_{i, 2 j}(i \neq 2 j)$ preserves areas with the coefficient $J\left(\Omega_{i, 2 j}\right)=16(j / i)^{4}$ and has the inverse mapping $\Omega_{i, 2 j}^{-1}=\Omega_{2 j, i}$. If $\left(\lambda_{1}, \mu_{1}\right) \in F_{1}^{ \pm}(\alpha)$ then the point $\left(4 j^{2} \lambda_{1}, 4 j^{2} \mu_{1}\right)(j \in \mathbb{N})$ is a cross point of the $\left(\frac{2 j}{i} \alpha\right)$-branch $\mathcal{F}_{2 j-1}^{ \pm}\left(\frac{2 j}{i} \alpha\right)$ and the line $\mu=\frac{\mu_{1}}{\lambda_{1}} \lambda$.

\section{Solution Set of a One-Parameter Nonlinear Oscillator}

Let us consider the problem (1.8), where the functions $f(x)=h^{+}(x)$, and $g(x)=h^{-}(-x)(x \geq 0)$ satisfy conditions (A1)-(A4). The problem (1.8) has been extensively studied in literature, overview of the theory can be found in $[10]$.

A solution set of the problem (1.8) is a set $G$ of all pairs $(\lambda, \alpha)$ such that there exists a nontrivial solution $x(t)$ of the problem (1.8) and the condition 
(1.4) fulfils. It follows from Theorem 4 that 1) the solution set $G$ of the problem (1.8) is a union of solution curves

$$
G_{i}^{ \pm}=\left\{(\lambda, \alpha): T_{f}(\alpha, \lambda)+T_{g}(\alpha, \lambda)=\frac{2(b-a)}{i}\right\} \quad(i \in \mathbb{N}) ;
$$

the solution curve $G_{i}^{+}\left(G_{i}^{-}\right)$describes all $C^{2}$-solutions of the problem (1.8) which have exactly $i$ zeros in the interval $(a, b)$ and negative (positive) derivative at the first after $t=a$ zero, $-\alpha$ and $\alpha$ respectively; 2) solution curves $G_{i}^{+}$and $G_{i}^{-}$coincide for any $i$, but solution curves $G_{i}^{ \pm}$and $G_{j}^{ \pm}$do not intersect if $i \neq j$.

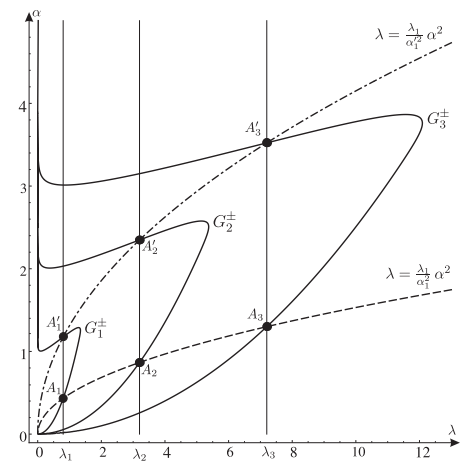

Figure 4. The solution curves $G_{j}^{ \pm} \quad(j=1,2,3)$ of the problem (1.8), where $f(x)=h^{+}(x)=x^{\frac{1}{2}}+x^{32}, g(x)=h^{-}(-x)=x^{\frac{1}{2}}+x^{32}, x \geq 0$ and $b-a=2.5$. The points $A_{j}\left(j^{2} \lambda_{1}, j \alpha_{1}\right) \in G_{j}^{ \pm}$and $A_{j}^{\prime}\left(j^{2} \lambda_{1}, j \alpha_{1}^{\prime}\right) \in G_{j}^{ \pm}$lie on the parabolas $\lambda=\frac{\lambda_{1}}{\alpha_{1}^{2}} \alpha^{2}$ and $\lambda=\frac{\lambda_{1}}{\alpha_{1}^{\prime 2}} \alpha^{2}$ respectively.

Using results on centro-affine mappings described in Theorems 4, 5 and Remarks 8, 9 we can supplement the theory of similarity of solution branches (solution curves) outlined in $[9,10,15]$.

Theorem 7. Consider the problem (1.8), where the functions $f(x)=h^{+}(x)$ and $g(x)=h^{-}(-x)(x \geq 0)$ satisfy the conditions (A1)-(A4).

1. For given $i \neq j$ the solution curves $G_{i}^{ \pm}$and $G_{j}^{ \pm}$are centro-affine equivalent under the mapping $\Gamma_{i, j}: \mathbb{R}^{2} \rightarrow \mathbb{R}^{2},(\lambda, \alpha) \stackrel{\Gamma_{i, j}}{\longmapsto}(\bar{\lambda}, \bar{\alpha})$, where $\bar{\lambda}=$ $\left(\frac{j}{i}\right)^{2} \lambda, \bar{\alpha}=\frac{j}{i} \alpha$.

2. For given $i \neq 2 j$ the solution curve $G_{i}^{ \pm}$of the Neumann problem (1.8) is centro-affine equivalent to the solution curve

$$
\mathcal{G}_{2 j-1}^{ \pm}=\left\{(\lambda, \alpha): T_{f}(\alpha, \lambda)+T_{g}(\alpha, \lambda)=\frac{b-a}{j}\right\}
$$

of the Dirichlet problem $x^{\prime \prime}=-\lambda h(x), x(a)=0=x(b)$ under the mapping $\Gamma_{i, 2 j}: \mathbb{R}^{2} \rightarrow \mathbb{R}^{2},(\lambda, \alpha) \stackrel{\Gamma_{i, 2 j}}{\longmapsto}(\widehat{\lambda}, \widehat{\alpha})$, where $\widehat{\lambda}=\left(\frac{2 j}{i}\right)^{2} \lambda, \widehat{\alpha}=\frac{2 j}{i} \alpha$. If $i=2 j$ then $G_{i}^{ \pm}=\mathcal{G}_{2 j-1}^{ \pm}\left(G_{2}^{ \pm}=\mathcal{G}_{1}^{ \pm}, G_{4}^{ \pm}=\mathcal{G}_{3}^{ \pm}, \ldots\right)$. 
Corollary 3. If $i \neq j$ the solution curve $\mathcal{G}_{2 i-1}^{ \pm}$is centro-affine equivalent to the solution curve $\mathcal{G}_{2 j-1}^{ \pm}$under the mapping $\Gamma_{i, j}$.

Remark 11. The analogue of the condition (1.4) in the Dirichlet problem is $\left|x^{\prime}(a)\right|=\alpha$. Solutions associated with the positive branch satisfy the condition $x^{\prime}(a)=\alpha>0$. Respectively, solutions associated with the negative branch satisfy the condition $x^{\prime}(a)=-\alpha<0$.

Remark 12. The centro-affine mapping $\Gamma_{i, j}(i \neq j)$ preserves areas with the coefficient $J\left(\Gamma_{i, j}\right)=(j / i)^{3}$ and has the inverse mapping $\Gamma_{i, j}^{-1}=\Gamma_{j, i}$. If $\left(\lambda_{1}, \alpha_{1}\right) \in G_{1}^{ \pm}$then the point $\left(j^{2} \lambda_{1}, j \alpha_{1}\right) \in G_{j}^{ \pm}(j \in \mathbb{N}, j \neq 1)$ is a cross point of the solution curve $G_{j}^{ \pm}$and the parabola $\lambda=\frac{\lambda_{1}}{\alpha_{1}^{2}} \alpha^{2}$.

Remark 13. The centro-affine mapping $\Gamma_{i, 2 j}(i \neq 2 j)$ preserves areas with the coefficient $J\left(\Gamma_{i, 2 j}\right)=8(j / i)^{3}$ and has the inverse mapping $\Gamma_{i, 2 j}^{-1}=\Gamma_{2 j, i}$. If $\left(\lambda_{1}, \alpha_{1}\right) \in G_{1}^{ \pm}$then the point $\left(4 j^{2} \lambda_{1}, 2 j \alpha_{1}\right)(j \in \mathbb{N})$ is a cross point of the solution curve $\mathcal{G}_{2 j-1}^{ \pm}$and the parabola $\lambda=\frac{\lambda_{1}}{\alpha_{1}^{2}} \alpha^{2}$.

Example 4. The solution curves $G_{j}^{ \pm}(j=1,2,3)$ of the problem (1.8), where $f=g=x^{1 / 3}+x^{32}$ and $b-a=2.5$, are centro-affine equivalent, as shown in Figure 4 .

Remark 14. Continuing Remark 6 notice that $i$-th $\alpha_{0}$-branch of the $\alpha_{0}$-spectrum of the $\alpha_{0}$-normalized one-parameter nonlinear problem (1.8), (1.5) actually can be identified with the cross section $G_{i}^{ \pm}\left(\alpha_{0}\right)$ of the solution curve $G_{i}^{ \pm}$and the level line $\alpha=\alpha_{0}$. The $\alpha_{0}$-branch $G_{i}^{ \pm}\left(\alpha_{0}\right)$ is a non empty set since due to the assertions 1 and 4 of Theorem 1 the function $T_{f}\left(\alpha_{0}, \lambda\right)+T_{g}\left(\alpha_{0}, \lambda\right)$ is continuous in $\lambda$ with the same limits as in (2.3), hence there exists $\lambda_{0}>0$ such that $T_{f}\left(\alpha_{0}, \lambda_{0}\right)+T_{g}\left(\alpha_{0}, \lambda_{0}\right)=2(b-a) / i$.

If $f=g$ then due to the rescaling formula (2.2) the solution curve

$$
G_{i}^{ \pm}=\left\{(\lambda, \alpha): T_{f}(\alpha, \lambda)=(b-a) / i\right\}
$$

can be parameterized without self intersections as follows: $\alpha=p_{i}(\gamma), \lambda=q_{i}(\gamma)$ $(\gamma>0)$, where $p_{i}(\gamma)=\frac{i \gamma t_{f}(\gamma)}{b-a}$ and $q_{i}(\gamma)=\left(\frac{i t_{f}(\gamma)}{b-a}\right)^{2}$.

Theorem 8. Consider the problem (1.8), where the functions $f(x)=h^{+}(x)$ and $g(x)=h^{-}(-x)(x \geq 0)$ satisfy the conditions (A1)-(A4). Suppose $f=g$ and the function $\gamma t_{f}(\gamma)$ has only two points of local extrema in the interval $(0,+\infty)$ : a point of strict maximum $\gamma_{\max }$ and strict minimum $\gamma_{\min }$, $0<\gamma_{\max }<\gamma_{\min }$. For given $\alpha_{0}>0$ and $i \in \mathbb{N}$ the $\alpha_{0}$-branch $G_{i}^{ \pm}\left(\alpha_{0}\right)$ of the $\alpha_{0}$-normalized problem (1.8), (1.5) is

- a one-point set if $0<\alpha_{0}<p_{i}\left(\gamma_{\min }\right)$ or $\alpha_{0}>p_{i}\left(\gamma_{\max }\right)$,

- a two-point set if $\alpha_{0}=p_{i}\left(\gamma_{\min }\right)$ or $\alpha_{0}=p_{i}\left(\gamma_{\max }\right)$,

- $a$ three-point set if $p_{i}\left(\gamma_{\min }\right)<\alpha_{0}<p_{i}\left(\gamma_{\max }\right)$. 
Proof. Taking into account (3.2) one has that the function $p_{i}(\gamma)=\frac{i \gamma t_{f}(\gamma)}{b-a}$ is strictly increasing in the intervals $I_{1}=\left(0, \gamma_{\max }\right], I_{3}=\left[\gamma_{\min },+\infty\right)$ and strictly decreasing in the interval $I_{2}=\left[\gamma_{\max }, \gamma_{\min }\right], p_{i}\left(I_{k}\right)=J_{i k}(k=1,2,3)$, $J_{i 1}=\left(0, p_{i}\left(\gamma_{\max }\right)\right], J_{i 2}=\left[p_{i}\left(\gamma_{\min }\right), p_{i}\left(\gamma_{\max }\right)\right]$ and $J_{i 3}=\left[p_{i}\left(\gamma_{\min }\right),+\infty\right)$. In the interval $J_{i k}(k=1,2,3)$ the formula $\lambda=\left(\alpha / p_{i}^{-1}(\alpha)\right)^{2}$ well defines a continuous function with the graph $\ell_{i, k}$ and the solution curve $G_{i}^{ \pm}$can be represented as the union of the graphs $\ell_{i, k}(k=1,2,3)$ which are continuously "glued" at the points $\left(q_{i}\left(\gamma_{\min }\right), p_{i}\left(\gamma_{\min }\right)\right)$ and $\left(q_{i}\left(\gamma_{\max }\right), p_{i}\left(\gamma_{\max }\right)\right)$. Since the parametrization $\alpha=p_{i}(\gamma), \lambda=q_{i}(\gamma)$ of the solution curve $G_{i}^{ \pm}$is without self intersections any two graphs $\ell_{i, k}(k=1,2,3)$ do not intersect except at may be "gluing" points. The theorem is proved.

Example 5. Depicted in Fig. 4 solution curves $G_{j}^{ \pm}(j=1,2,3)$ illustrate Theorem 8 .

Table 1. Centro-affine equivalence formulae for the Neumann and Dirichlet BVP.

\begin{tabular}{|c|c|}
\hline$x^{\prime}(a)=0=x^{\prime}(b)(*) \mid x(a)=0=x(b)(* *)$ & $(*) \rightarrow(* *)$ \\
\hline \multicolumn{2}{|c|}{$\begin{array}{l}\text { Centro-affine equivalence of solution surfaces of } \\
\text { the two-parameter nonlinear oscillator } x^{\prime \prime}=-\lambda f\left(x^{+}\right)+\mu g\left(x^{-}\right) \text {, } \\
\qquad\left|x^{\prime}(z)\right|=\alpha \text { at zeroes of a solution } x(t)\end{array}$} \\
\hline $\begin{array}{c}(\lambda, \mu, \alpha) \stackrel{\Phi_{i, j}}{\longmapsto}(\bar{\lambda}, \bar{\mu}, \bar{\alpha}), i \neq j \\
\bar{\lambda}=\left(\frac{j}{i}\right)^{2} \lambda, \bar{\mu}=\left(\frac{j}{i}\right)^{2} \mu, \bar{\alpha}=\frac{j}{i} \alpha \\
J\left(\Phi_{i, j}\right)=\left(\frac{j}{i}\right)^{5}, \Phi_{i, j}^{-1}=\Phi_{j, i} \\
\Phi_{i, j}\left(F_{i}^{ \pm}\right)=F_{j}^{ \pm} \quad \mid \Phi_{i, j}\left(\mathcal{F}_{2 i-1}^{ \pm}\right)=\mathcal{F}_{2 j-1}^{ \pm}\end{array}$ & $\begin{array}{c}(\lambda, \mu, \alpha) \stackrel{\Phi_{i, 2 j}}{\longmapsto}(\widehat{\lambda}, \widehat{\mu}, \widehat{\alpha}), i \neq 2 j \\
\widehat{\lambda}=\left(\frac{2 j}{i}\right)^{2} \lambda, \widehat{\mu}=\left(\frac{2 j}{i}\right)^{2} \mu, \widehat{\alpha}=\frac{2 j}{i} \alpha \\
J\left(\Phi_{i, 2 j}\right)=32\left(\frac{j}{i}\right)^{5}, \Phi_{i, 2 j}^{-1}=\Phi_{2 j, i} \\
\Phi_{i, 2 j}\left(F_{i}^{ \pm}\right)=\mathcal{F}_{2 j-1}^{ \pm}\end{array}$ \\
\hline \multicolumn{2}{|c|}{$\begin{array}{l}\text { Centro-affine equivalence of branches of the } \alpha \text {-normalized } \\
\text { problem } x^{\prime \prime}=-\lambda f\left(x^{+}\right)+\mu g\left(x^{-}\right) \\
\qquad\left|x^{\prime}(z)\right|=\alpha \text { at zeroes of a solution } x(t), \alpha \text { - fixed }\end{array}$} \\
\hline 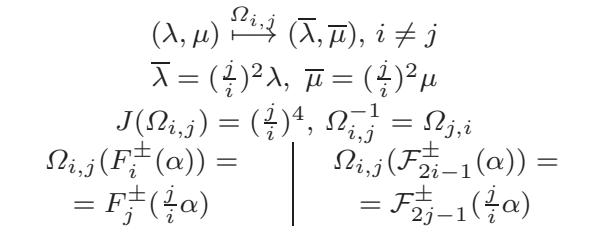 & $\begin{array}{c}(\lambda, \mu) \stackrel{\Omega_{i, 2 j}}{\longmapsto}(\widehat{\lambda}, \widehat{\mu}), i \neq 2 j \\
\widehat{\lambda}=\left(\frac{2 j}{i}\right)^{2} \lambda, \widehat{\mu}=\left(\frac{2 j}{i}\right)^{2} \mu \\
J\left(\Omega_{i, 2 j}\right)=16\left(\frac{j}{i}\right)^{4}, \Omega_{i, 2 j}^{-1}=\Omega_{2 j, i} \\
\Omega_{i, 2 j}\left(F_{i}^{ \pm}(\alpha)\right)= \\
=\mathcal{F}_{2 j-1}^{ \pm}\left(\frac{2 j}{i} \alpha\right)\end{array}$ \\
\hline
\end{tabular}

Centro-affine equivalence of solution curves of

the one-parameter nonlinear oscillator $x^{\prime \prime}=-\lambda f\left(x^{+}\right)+\lambda g\left(x^{-}\right)$, $\left|x^{\prime}(z)\right|=\alpha$ at zeroes of a solution $x(t)$

\begin{tabular}{c|c}
\hline$(\lambda, \alpha) \stackrel{\Gamma_{i, j}}{\longmapsto}(\bar{\lambda}, \bar{\alpha}), i \neq j$ & $(\lambda, \alpha) \stackrel{\Gamma_{i, 2 j}}{\longmapsto}(\widehat{\lambda}, \widehat{\alpha}), i \neq 2 j$ \\
$\bar{\lambda}=\left(\frac{j}{i}\right)^{2} \lambda, \bar{\alpha}=\frac{j}{i} \alpha$ & $\widehat{\lambda}=\left(\frac{2 j}{i}\right)^{2} \lambda, \widehat{\alpha}=\frac{2 j}{i} \alpha$ \\
$J\left(\Gamma_{i, j}\right)=\left(\frac{j}{i}\right)^{3}, \Gamma_{i, j}^{-1}=\Gamma_{j, i}$ & $J\left(\Gamma_{i, 2 j}\right)=8\left(\frac{j}{i}\right)^{3}, \Gamma_{i, 2 j}^{-1}=\Gamma_{2 j, i}$ \\
$\Gamma_{i, j}\left(G_{i}^{ \pm}\right)=G_{j}^{ \pm}$ & $\Gamma_{i, j}\left(\mathcal{G}_{2 i-1}^{ \pm}\right)=\mathcal{G}_{2 j-1}^{ \pm}$
\end{tabular}




\section{Conclusions}

A two-parameter nonlinear oscillator with the Neumann boundary conditions exhibits the following features.

- $\alpha$-spectrum is similar to the classical Fučík spectrum if the functions $T_{f}(\alpha, \lambda)$ and $T_{g}(\alpha, \mu)$ are monotone in $\lambda$ and $\mu$ respectively.

- Otherwise it is possible that $\alpha$-branches of an $\alpha$-spectrum have separate bounded components.

- Different branches $F_{i}^{ \pm}(\alpha)$ and $F_{j}^{ \pm}(\beta)$ of spectra with different normalizations may intersect (even coincide). This means that oscillations near cross-points $(\lambda, \mu)$ may switch instantly from one nodal type to another one. This behavior was recognized for large asymmetrical oscillators such as suspension bridges [11, p. 540]. The branches may not intersect as well.

- A solution set is a union of solution surfaces $F_{i}^{ \pm}$, besides any two solution surfaces $F_{i}^{ \pm}$and $F_{j}^{ \pm}(i \neq j)$ are centro-affine equivalent. For given $i \neq 2 j$ the solution surface $F_{i}^{ \pm}$is centro-affine equivalent to the solution surface $\mathcal{F}_{2 j-1}^{ \pm}$for the Dirichlet problem. In other words these solution surfaces have the same shape.

- The technique of affine mappings can be applied to the special cases of a two-parameter nonlinear oscillator also - $\alpha$-normalized problem and oneparameter nonlinear problem. In Table 1, the respective centro-affine equivalence formulae are collected.

\section{References}

[1] C. De Coster and P. Habets. A two parameters Ambrosetti-Prodi problem. Port. Math., 53(3):279-303, 1996.

[2] W. Dambrosio. Qualitative results for parameter dependent asymmetric oscillators. Nonlinear Anal., 47(7):5021-5028, 2001.

Doi:10.1016/S0362-546X(01)00613-7.

[3] S. Fučík and A. Kufner. Nonlinear Differential Equations. Elsevier, 1980.

[4] A. Gritsans and F. Sadyrbaev. Nonlinear spectra for parameter dependent ordinary differential equations. Nonlinear Anal. Model. Control, 12(2):253-267, 2007.

[5] A. Gritsans and F. Sadyrbaev. On nonlinear Fučík type spectra. Math. Model. Anal., 13(2):203-210, 2008. Doi:10.3846/1392-6292.2008.13.203-210.

[6] A. Gritsans and F. Sadyrbaev. Time map formulae and their applications. In Proceedings LU MII "Mathematics. Differential Equations", Vol. 8, pp. 72-93, Riga, 2008.

[7] A. Gritsans and F. Sadyrbaev. Two-parametric nonlinear eigenvalue problems. In L. Hatvani and T. Krisztin(Eds.), Proc. of the 8'th Colloquium on the Qualitative Theory of Differential Equations, Szeged, Hungary, 2007, pp. 1-14, 2008. 
[8] A. Gritsans and F. Sadyrbaev. Nonlinear spectra: the Neumann problem. Math. Model. Anal., 14(1):33-42, 2009. Doi:10.3846/1392-6292.2009.14.33-42.

[9] P. Korman. Similarity of solution branches for two-point semilinear problems. In Electron. J. Differ. Equ., Conf. 10, pp. 187-191, 2003.

[10] P. Korman. Global solution branches and exact multiplicity of solutions for two point boundary value problems. In A. Canada, P. Drabek and A. Fonda(Eds.), Handbook of Differential Equations, Ordinary Differential Equations, Vol. 3, pp. 547-606. Elsevier Science, North-Holland, Amsterdam, 2006.

[11] A.C. Lazer and P.J. McKenna. Large-amplitude periodic oscillations in suspension bridges: Some new connections with nonlinear analysis. SIAM Rev., 32(4):537-578, 1990. Doi:10.1137/1032120.

[12] Z. Opial. Sur les périodes des solutions de l'équation différentielle $x^{\prime \prime}+g(x)=0$. Ann. Polon. Math., 10:49-72, 1961.

[13] B. Rosenfeld. Multidimensional Spaces. Nauka, 1966. (in Russian)

[14] F. Sadyrbaev. Multiplicity in parameter-dependent problems for ordinary differential equations. Math. Model. Anal., 14(4):503-514, 2009. Doi:10.3846/1392-6292.2009.14.503-514.

[15] R. Schaaf. Global solution branches of two point boundary value problems, volume 1458 of Lecture Notes in Math. Springer, 1990.

[16] T. Shibata. Two-parameter nonlinear Sturm-Liouville problems. Proc. Edinb. Math. Soc., 41(2):225-245, 1998. Doi:10.1017/S0013091500019611. 